\title{
Energetically Consistent Boundary Conditions for Electromechanical Fracture
}

\author{
Chad M. Landis \\ Department of Mechanical Engineering and Materials Science, MS 321 \\ Rice University, P.O. Box 1892 \\ Houston, TX 77251-1892 \\ Phone: (713) 348-3609, Fax: (713) 348-5423 \\ E-mail: landis@rice.edu
}

\begin{abstract}
Energetically consistent crack face boundary conditions are presented for cracks in electromechanical materials. The model assumes that the energy of the solid can be computed from standard infinitesimal deformation theory and that the opening of the crack faces creates a capacitive gap that can store electrical energy. The general derivation of the crack face boundary conditions is carried out for nonlinear but reversible constitutive behavior of both the solid material and the space filling the gap. It is shown that a simple augmentation of the $J$-integral can be used to determine the energy release rate for crack advance with these boundary conditions. The energetically consistent boundary conditions are then applied to the Griffith crack problem in a polar linear piezoelectric solid and used to demonstrate that the crack tip energy release rate is equivalent to the global energy release rate for the system. A nonlinear constitutive law is postulated for the crack gap as a model for electrical discharge and the effects of the breakdown field on the energy release rate are ascertained.
\end{abstract}

Keywords: piezoelectricity, polarized material, electrical discharge, Griffith crack, boundary conditions, energy release rates

\section{Background}

This work reports on results that have been developed in greater detail in Landis (2004). The purpose of this work is to investigate energetically consistent forms of crack face boundary conditions for fracture in electromechanically active materials.

In order to determine the consistent boundary conditions for a crack gap that is able to support electrical fields the following are implemented. (1) Linear kinematics can be used to describe the deformation of the cracked solid. (2) The energy of the cracked body can be computed using its undeformed configuration. (3) When the crack opens electric fields can permeate the crack medium and electrical energy can be stored within the crack. (4) The energy stored within the crack medium can be computed from the deformed configuration of the cracked body. (5) Electric field components within the crack medium parallel to the crack faces are negligible compared to the electric field normal to the crack.

The energetically consistent boundary conditions are then determined from the following procedure. First, the variation of the total electrical enthalpy of a combined cracked solid and crack gap system is derived. Then, a second system is proposed with the crack gap removed, and in its place tractions and surface charge densities are applied to the crack surfaces. In order for these two systems to be equivalent, the variations of the total electrical enthalpy of these systems must be identical for arbitrary variations of the crack face displacement and electric potential. Applying these identities will allow for the identification of effective tractions and surface charge densities that are applied by the crack gap medium to the cracked body.

The total electrical enthalpy $\Omega$ of the solid-crack system can then be written as, Suo et al. (1992),

$$
\Omega(\mathbf{u}, \phi)=\int_{V} h \mathrm{~d} V+\int_{S_{c}} h_{c} \Delta u_{n} \mathrm{~d} S-\int_{V} b_{i} u_{i} \mathrm{~d} V+\int_{V} q \phi \mathrm{d} V-\int_{S_{t}} t_{i} u_{i} \mathrm{~d} S+\int_{S_{\omega}} \omega \phi \mathrm{d} S
$$

Here $\mathbf{u}$ is the displacement vector with Cartesian components $u_{i}, \phi$ is the electric potential, $h$ is the electrical enthalpy density of the solid, $h_{c}$ is the electrical enthalpy density of the crack gap, $\Delta u_{n}$ is the opening displacement of the crack, $b_{i}$ the components of the applied body force, $q$ the applied volume charge density, $t_{i}$ the components of the surface traction, $\omega$ the applied surface charge, $S_{c}$ is the crack surface, $S_{t}$ the region of the material surface where tractions are applied, and $S_{\omega}$ the region of the surface where charges are applied. Here the second term on the right hand side has been included to account for the electrical enthalpy of the crack gap that has an electrical enthalpy density of $h_{c}$. Note that it is assumed that the separation of the crack surfaces is small such that the volume of the crack gap is given by $V_{c}=\Delta u_{n} S_{c}$. In essence, the evaluation of this second term is carried out in the deformed configuration of the body while the remaining terms are evaluated in the 
undeformed configuration. Herein lies a fundamental inconsistency with this model that can only be properly removed with a more general large deformation analysis. However, the assumptions associated with Equation (1.1) are entirely consistent with the "exact" boundary conditions proposed by Hao and Shen (1994) that appear extensively in the literature.

Applying the following definitions,

$$
E_{c}=-\frac{\Delta \phi}{\Delta u_{n}} \text { and } D_{c}=-\frac{\partial h_{c}}{\partial E_{c}}
$$

with $\Delta \phi$ being the electric potential jump across the crack, the variation of the second term of Equation (1.1) can be written as

$$
\int_{S_{c}}\left(\Delta u_{n} \frac{\partial h_{c}}{\partial E_{c}} \delta E_{c}+h_{c} \delta \Delta u_{n}\right) \mathrm{d} S=\int_{S_{c}}\left[D_{c} \delta \Delta \phi+\left(h_{c}+D_{c} E_{c}\right) \delta \Delta u_{n}\right] \mathrm{d} S
$$

Recall that this term represents the variation of the contribution to the total electrical enthalpy from the crack. This term consists of an electrical contribution associated with the electric field acting through the crack gap plus a mechanical contribution arising from the fact that the stored electrical energy within the crack gap increases as the volume of the gap increases. Hence, there is an increase in energy of the system associated with increasing crack opening displacement, and the work conjugate force for this configurational change is equivalent to the internal energy density of the crack gap, i.e. $h_{c}+E_{c} D_{c}=\tilde{u}_{c}$. This work conjugate force will be renamed $\sigma_{c}$ or the effective stress within the crack gap. Such stresses that occur due to displacements and electrical effects are common in more general studies on large deformation behavior of electrically active materials and are referred to as Maxwell stresses.

Ultimately, it is shown in Landis (2004) that the general energetically consistent boundary conditions on the crack faces can be derived as,

$$
\begin{aligned}
& \omega^{+}=-D_{i} n_{i}^{+}=D_{c}=-\frac{\partial h_{c}}{\partial E_{c}} \text { on } S_{c}^{+} \\
& \omega^{-}=-D_{i} n_{i}^{-}=-D_{c}=\frac{\partial h_{c}}{\partial E_{c}} \text { on } S_{c}^{-} \\
& t_{i}^{+}=\sigma_{j i} n_{j}^{+}=\sigma_{c} n_{i}^{+}=\left(h_{c}+E_{c} D_{c}\right) n_{i}^{+} \text {on } S_{c}^{+} \\
& t_{i}^{-}=\sigma_{j i} n_{j}^{-}=\sigma_{c} n_{i}^{-}=\left(h_{c}+E_{c} D_{c}\right) n_{i}^{-} \text {on } S_{c}^{-}
\end{aligned}
$$

Here the superscript + or - represents the top or bottom crack face and the $n_{i}$ are the components of the associated unit normal pointing into the crack. In the following, more specific forms for these boundary conditions will be given for the special cases of perfect linear dielectric crack gap behavior and an idealized model for electrical discharge within the crack gap.

\section{The linear dielectric and discharging crack gaps}

Consider a crack gap with linear dielectric behavior that has been studied by numerous authors. Specifically, the electric field versus electric displacement behavior of the gap is assumed to be

$$
D_{c}=\kappa_{0} E_{c}
$$

Here $\kappa_{0}$ is the dielectric constant of the material filling the crack gap and in most situations is identified as the dielectric permittivity of free space. Then, the electrical enthalpy density of the crack gap for this case is

$$
h_{c}=-\frac{1}{2} \kappa_{0} E_{c}^{2}
$$

Finally, the crack boundary conditions can be stated as 


$$
D_{n}^{+}=D_{n}^{-}=-\kappa_{0} \frac{\phi^{+}-\phi^{-}}{u_{n}^{+}-u_{n}^{-}} \text {on } S_{c}^{+} \text {and } S_{c}^{-}
$$

and

$$
\sigma_{n n}^{+}=\sigma_{n n}^{-}=\frac{1}{2} \kappa_{0}\left(\frac{\phi^{+}-\phi^{-}}{u_{n}^{+}-u_{n}^{-}}\right)^{2} \text { on } S_{c}^{+} \text {and } S_{c}^{-}
$$

Here a convention is defined on the crack faces such that the subscript $n$ represents the component normal to the crack surfaces with the positive direction associated with the lower crack face normal. Specifically, $D_{n}^{+}=D_{i}^{+} n_{i}^{-}, D_{n}^{-}=D_{i}^{-} n_{i}^{-}, u_{n}^{+}=u_{i}^{+} n_{i}^{-}$, $u_{n}^{-}=u_{i}^{-} n_{i}^{-}, \sigma_{n n}^{+}=\sigma_{j i}^{+} n_{j}^{-} n_{i}^{-}$and $\sigma_{n n}^{-}=\sigma_{j i}^{-} n_{j}^{-} n_{i}^{-}$(no summation over $n$ ). Note that for the standard crack configuration with the crack lying along the $x_{1}$-axis and perpendicular to the $x_{2}$-axis, the subscript $n$ in Equations (2.3) and (2.4) can be replaced by a numeral 2. Also note that the electric displacements and stresses denoted in these equations are those quantities in the solid at the crack surface. Finally, Equation (2.3) is the so-called "exact" boundary condition that has received considerable study in the literature. However, aside from the work of Landis and McMeeking (2000), the non-zero traction component of these boundary conditions has yet to be recognized or studied.

For the electrically discharging crack gap it is assumed that the crack gap behaves in a linear dielectric fashion up to some critical electric field level for discharge $E_{d}$. At this point the electric displacement in the gap will be $D_{c}=\kappa_{0} E_{d}$. It will be assumed that the crack gap cannot support electric fields larger than $E_{d}$, but that charge can be transferred between the crack faces such that the effective electric displacement of the crack gap can increase without bound.

For this simple model for discharge, the electric displacement in the crack gap takes on the mathematical form,

$$
\begin{aligned}
& D_{c}=\kappa_{0} E_{c} \text { if }\left|D_{c}\right| \leq \kappa_{0} E_{d} \\
& D_{c}=\operatorname{sgn}\left(\omega_{d}\right) \kappa_{0} E_{d}+\omega_{d} \text { and } E_{c}=\operatorname{sgn}\left(\omega_{d}\right) E_{d} \text { if }\left|D_{c}\right| \geq \kappa_{0} E_{d}
\end{aligned}
$$

Here $\omega_{d}$ represents the amount of charge per unit area transferred between the crack faces. Then, the electrical enthalpy density of the crack gap can be given as

$$
\begin{aligned}
& h_{c}=-\frac{1}{2} \kappa_{0} E_{c}^{2} \text { if }\left|D_{c}\right| \leq \kappa_{0} E_{d} \\
& h_{c}=-\frac{1}{2} \kappa_{0} E_{d}^{2} \text { if }\left|D_{c}\right| \geq \kappa_{0} E_{d}
\end{aligned}
$$

Then if $\left|D_{c}\right| \leq \kappa_{0} E_{d}$ the boundary conditions along the crack faces can be given as

$$
D_{n}^{+}=D_{n}^{-}=-\kappa_{0} \frac{\phi^{+}-\phi^{-}}{u_{n}^{+}-u_{n}^{-}} \text {on } S_{c}^{+} \text {and } S_{c}^{-}
$$

and

$$
\sigma_{n n}^{+}=\sigma_{n n}^{-}=\frac{1}{2} \kappa_{0}\left(\frac{\phi^{+}-\phi^{-}}{u_{n}^{+}-u_{n}^{-}}\right)^{2} \text { on } S_{c}^{+} \text {and } S_{c}^{-}
$$

If $\left|D_{c}\right| \geq \kappa_{0} E_{d}$ the boundary conditions are 


$$
\begin{aligned}
& -\frac{\phi^{+}-\phi^{-}}{u_{n}^{+}-u_{n}^{-}}=\operatorname{sgn}\left(D_{c}\right) E_{d} \text { on } S_{c}^{+} \text {and } S_{c}^{-} \\
& D_{n}^{+}=D_{n}^{-}=\kappa_{0} \operatorname{sgn}\left(D_{c}\right) E_{d}+\omega_{d} \text { on } S_{c}^{+} \text {and } S_{c}^{-}
\end{aligned}
$$

and

$$
\sigma_{n n}^{+}=\sigma_{n n}^{-}=E_{d}\left|D_{c}\right|-\frac{1}{2} \kappa_{0} E_{d}^{2}=E_{d}\left|\omega_{d}\right|+\frac{1}{2} \kappa_{0} E_{d}^{2} \text { on } S_{c}^{+} \text {and } S_{c}^{-}
$$

These boundary conditions will be used to analyze a Griffith crack in a linear piezoelectric solid in the next section.

\section{The Griffith crack in a poled linear piezoelectric solid}

In this section the energetically consistent boundary conditions will be applied to the Griffith crack in a poled linear piezoelectric solid. For simplicity the material will be poled perpendicular to the crack and only Mode I and Mode D loadings will be applied to the sample. For these calculations $D$ is the total electric displacement including the remanent polarization, $\omega_{s}$ is the charge separation along the crack faces, and $H_{11}$ and $H_{22}$ are components of the Irwin matrix. Details and descriptions of these parameters and the solution are given in Landis (2004). Specific cases will be solved and compared to ascertain the effects of the energetically consistent boundary conditions and especially the effects of electrical discharge within the crack.

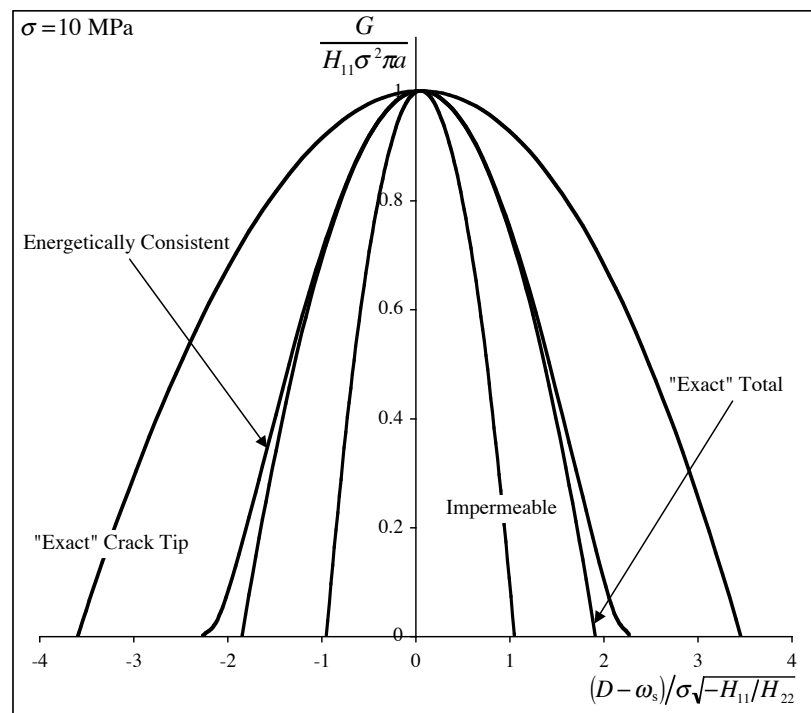

Figure 1. Energy release rates for a Griffith crack in a linear piezoelectric solid. The properties used for these calculations are those reported for PZT-5H. These results are valid for both polar and non-polar materials.

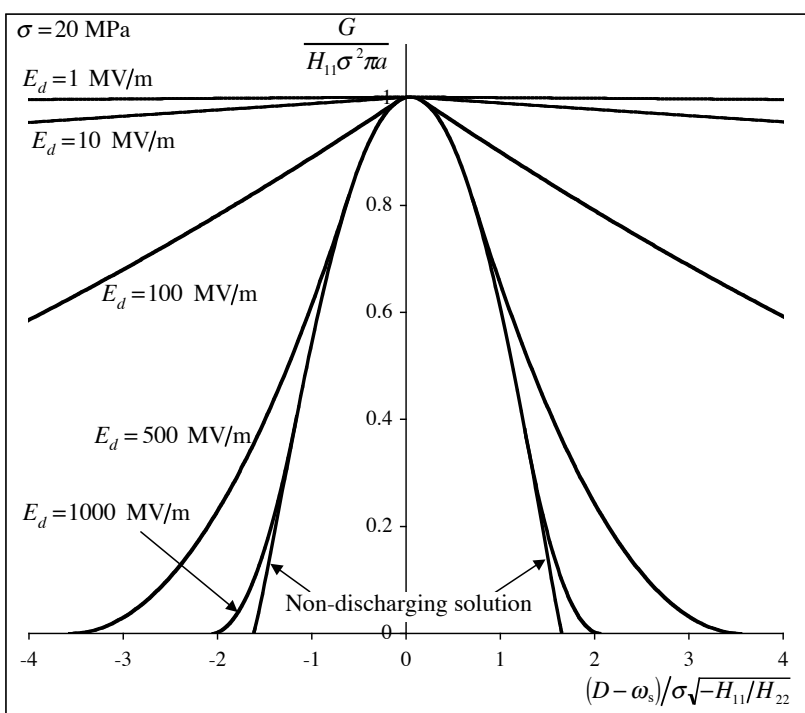

Figure 2. The effects of electric discharge on the energy release rate using the energetically consistent boundary conditions.

First, for comparison to the impermeable and "exact" boundary conditions appearing in the literature it will be assumed that the discharge field is extremely large such that $E_{d} \rightarrow \infty$. Figure 1 plots results for the energy release rates for all three boundary condition types. The values for the material properties used to generate the results shown in Figure 1 are characteristic of PZT$5 \mathrm{H}$. Note that the total and crack tip energy release rates are equal for the impermeable and energetically consistent boundary conditions, but these two quantities differ for the "exact" boundary conditions. Figure 1 illustrates a number of interesting features of this problem. First, for the modest applied stress level of 10-MPa the difference between the crack tip energy release rate and the total energy release rate for the "exact" boundary conditions that are so prevalent in the literature is significant over a wide range of $D-\omega_{\mathrm{s}}$. Furthermore, the simple fact that these two quantities differ, regardless of the magnitude of the difference, is unappealing from a theoretical perspective. It is also interesting to note that both the "exact" and energetically consistent boundary conditions yield energy release rates significantly higher than the energy release rate for the impermeable boundary conditions. This feature arises because the existence of cracks in the presence of electric fields tends to be a high-energy state and hence electric fields tend to retard crack growth and the energy released during crack 
growth. This retardation process is maximized for the impermeable boundary conditions, but reduced for the "exact" and energetically consistent boundary conditions where electric fields can permeate through the crack gap.

One final observation from the solutions presented in Figure 1 is that the electric field in the crack gap is much larger than the level of electric field applied to the solid. This feature of the solution, along with experimental observations of discharge in crack gaps, is the motivation to study the effects of electrical discharge on the energy release rate for this system. Figure 2 plots the results for the energy release rate versus the applied electric displacement for the energetically consistent boundary conditions for an applied stress level of $20-\mathrm{MPa}$ and various levels of the discharge field $E_{d}$. The most significant observation illustrated on Figure 2 is that electric discharge tends to reduce the retarding effects of electric field, thereby increasing the energy release rate. Also, note on Figure 2 that solutions for the energy release rate during electrical discharge drop smoothly and continuously to zero.

\section{Discussion}

This work has been motivated primarily by McMeeking's observation, McMeeking (2004), that the so-called "exact" electrical boundary conditions that are prevalent in the literature give rise to a discrepancy between the total and crack tip energy release rates in a cracked piezoelectric body. Such a discrepancy is objectionable from a theoretical perspective. To address this problem, energetically consistent electromechanical boundary conditions for cracks have been derived. These boundary conditions were derived based on the following assumptions. (1) The energy of the cracked body can be computed using its undeformed configuration. (2) When the crack opens electric fields can permeate the crack medium and electrical energy can be stored within the crack. (3) The energy stored within the crack medium can be computed from the deformed configuration of the cracked body. (4) Electric field components within the crack medium parallel to the crack faces are negligible compared to the electric field normal to the crack. Evidently, assumptions (1) and (3) are in contradiction with one another since the analysis of energies is mixed between deformed and undeformed configurations. However, these assumptions are consistent with those used for the "exact" boundary conditions. Furthermore, a proper resolution to this inconsistency would require a full nonlinear, large deformation kinematics analysis of the problem. Instead, in this work, assumptions (1)-(4) are taken as a starting point and the energetically consistent crack boundary conditions are derived by equating the weak statements of two boundary value problems; one which models the volume of the crack gap explicitly and one that models the crack gap through the surface tractions and charges that it applies to the cracked solid.

The primary results of this paper are the energetically consistent boundary conditions given by Equations (1.4)-(1.7). These boundary conditions imply that cracks in electromechanical materials not only sustain electric field and electric displacement, but also apply mechanical traction to the surrounding material. This feature of mechanical forces arising due to electrical effects is common in finite deformation analyses of electromechanical materials and is usually termed a Maxwell stress. The specific example of a Griffith crack in a poled linear piezoelectric solid was used to demonstrate that the energetically consistent boundary conditions do in fact resolve the discrepancy between the crack tip and total energy release rates. Furthermore, the effects of electrical discharge on the energy release rate was ascertained and shown to reduce the retarding effects of electric fields on crack growth.

\section{Acknowledgement}

The author would like to acknowledge support for this work from the National Science Foundation through grant number CMS-0238522.

\section{References}

Hao, T.-H., Shen, Z.-Y., 1994. A new electric boundary condition of electric fracture mechanics and its application. Eng. Fract. Mech. 47, 793-802.

Landis, C.M., McMeeking, R.M., 2000. Modeling of fracture in ferroelectric ceramics. Proceedings of the SPIE 3992, $176-184$.

Landis, C.M., 2004. Energetically consistent boundary conditions for electromechanical fracture. Int. J. Solids Struct., in press.

McMeeking, R.M., 2004. The energy release rate for a Griffith crack in a piezoelectric material. Eng. Fract. Mech. 71, 11491163.

Suo, Z., Kuo, C.M., Barnett, D.M., Willis, J.R., 1992. Fracture mechanics for piezoelectric ceramics. J. Mech. Phys. Solids 40, 739-765. 